\title{
Mixed-parity superconductivity near Lifshitz transitions in strongly spin-orbit-coupled metals
}

\author{
Matthew J. Trott $\odot$ and Chris A. Hooley $\odot$ \\ SUPA, School of Physics and Astronomy, University of St Andrews, North Haugh, St Andrews, Fife KY16 9SS, United Kingdom
}

(Received 4 October 2019; revised manuscript received 27 November 2019; published 31 January 2020)

\begin{abstract}
We consider a strongly spin-orbit-coupled metal, one of whose Fermi surfaces is close to a Lifshitz (topological) transition. Via a renormalization group analysis of the square-lattice Hubbard model with strong Rashba spin-orbit coupling, we show that such a metal is generically unstable to the formation of mixed-parity superconductivity with a helical triplet component.
\end{abstract}

DOI: 10.1103/PhysRevResearch.2.013106

\section{INTRODUCTION}

Topological superconductivity is at the forefront of modern investigations in materials physics due in part to its potential for realizing topological quantum computation via localized Majorana zero modes [1]. In order to obtain nontrivial topology, the superconductivity must be of an unconventional form, with spin-triplet Cooper pairs carrying nonzero angular momentum [2]. Such unconventional superconductivity is thought to arise from spin-fluctuation-mediated pairing, distinct from the phonon-mediated mechanism which is found in the majority of superconducting materials [3].

The search for materials that have the required topological characteristics is ongoing. A class of materials of great interest are those with noncentrosymmetric or nonsymmorphic crystal structures. The broken inversion symmetry of the noncentrosymmetric materials allows for antisymmetric spin-orbit coupling [4]. This leads to a mixing of spin-singlet and spintriplet Cooper pairs [5]. The noncentrosymmetric material $\mathrm{CePt}_{3} \mathrm{Si}$ is a candidate for an $(s+p)$-wave superconducting state $[6,7]$. It is also believed that $\mathrm{Li}_{2} \mathrm{Pt}_{3} \mathrm{~B}$ exhibits spin-triplet superconductivity [8].

Other spin-triplet candidates include cuprate thin films grown on a substrate. These films have an induced Rashba spin-orbit interaction which leads to a $(d+p)$-wave pairing state $[9,10]$. Unconventional superconductivity is also thought to arise at oxide interfaces [11]. Additionally, there are proposals to engineer unconventional superconductors via superlattices of organic molecules on superconducting substrates [12].

The unifying physics within the proposed mixed-parity superconductivity materials is the presence of antisymmetric spin-orbit coupling. This coupling induces spin flips during scattering processes, altering how the superconductivity is formed, and leads to an enhancement of the triplet component of the superconducting order parameter [13].

Published by the American Physical Society under the terms of the Creative Commons Attribution 4.0 International license. Further distribution of this work must maintain attribution to the author(s) and the published article's title, journal citation, and DOI.
A simple model that captures both the spin-orbit interaction and the electron-electron repulsion needed for unconventional pairing is the square-lattice Hubbard model with an additional Rashba spin-orbit coupling term. The Rashba term splits the underlying tight-binding band into two bands with nontrivial spin textures. This model, known as the Rashba-Hubbard model, has been investigated in several previous studies. Early analysis of the extended Rashba-Hubbard model using the random phase approximation (RPA) suggested mided-parity superconductivity $[13,14]$. Later RPA studies found regions of $d$-wave and $f$-wave superconductivity dominating $[15,16]$. Dynamical mean-fieldtheory studies found a mixed-parity state of $(d+p)$-wave superconductivity [17].

An important feature of the two-dimensional Hubbard model is the presence of saddle points in the tight-binding dispersion. The saddle points lead to a van Hove singularity that occurs when the system is doped through a Lifshitz transition, a Fermi surface transition between an open and closed Fermi surface, or the connection of two or more Fermi surface pockets $[18,19]$. At a van Hove singularity, the density of states diverges logarithmically, with electrons around saddle points in the dispersion giving rise to the divergence. A patch approximation can then be constructed around the van Hove saddle points to examine the possible Fermi surface instabilities of the system [20-22].

In the Rashba-Hubbard model, there are two filling fractions at which the Fermi surface touches the van Hove points. At each such filling, all the van Hove points lie on one Fermi surface sheet such that the predominant contribution to the susceptibilities comes from a single helicity band in the lowenergy limit.

When electron-electron interactions are stronger than or comparable to the Rashba coupling, significant scattering between the two helicity bands is expected. However, when the electron-electron interactions are much weaker than the Rashba coupling, low-energy scattering is predominantly intraband, and that is the limit we shall consider here.

In this paper, following [23-25], we construct a patch renormalization group ( $R G)$ scheme applicable near the two van Hove fillings of the Rashba-Hubbard model. In the limit of weak coupling, we perform a one-loop RG to find the leading instability of the system. We show that mixed-parity 
superconductivity arises naturally from this scheme, demonstrating the importance of the spin helicity structure when considering spin-orbit coupled materials.

\section{MODEL AND METHODS}

We consider a square lattice tight-binding model,

$$
\begin{aligned}
H_{0}= & -t \sum_{\langle i, j\rangle, s} c_{i s}^{\dagger} c_{j s}-t^{\prime} \sum_{\langle i, j\rangle, s} c_{i s}^{\dagger} c_{j s}-\mu \sum_{i, s} c_{i s}^{\dagger} c_{i s} \\
& +i v \sum_{\langle i, j\rangle, s, s^{\prime}}\left[\left(\sigma \times \mathbf{a}_{i j}\right) \cdot \hat{\mathbf{z}}\right]_{s s^{\prime}} c_{i s}^{\dagger} c_{j s^{\prime}} .
\end{aligned}
$$

$\langle i, j\rangle$ and $\langle\langle i, j\rangle\rangle$ denote nearest-neighbor and next-nearestneighbor hopping with hopping strengths $t$ and $t^{\prime}$, respectively. The spin orientations are denoted $s, s^{\prime} \in\{\uparrow, \downarrow\} . v$ is the Rashba spin-orbit coupling strength, $\sigma=\left(\sigma_{x}, \sigma_{y}, \sigma_{z}\right)^{T}$ the vector of Pauli matrices, and $\mathbf{a}_{i j}$ denotes the unit vectors between nearest-neighbor sites.

The Hubbard interaction term is

$$
V_{\mathrm{int}}=\frac{U}{2} \sum_{s, s^{\prime}} \sum_{k_{1} k_{2} k_{3} k_{4}} \delta_{k_{1}+k_{2}-k_{3}-k_{4}} c_{k_{1} s}^{\dagger} c_{k_{2} s^{\prime}}^{\dagger} c_{k_{3} s^{\prime}} c_{k_{4} s},
$$

describing a contact interaction which is repulsive for $U>0$ and attractive for $U<0$. The interacting Hamiltonian $H$ is given by $H_{0}+V_{\text {int }}$.

Spin-orbit coupling breaks the spin degeneracy of the noninteracting bands and splits them into two with opposite helicities. After a unitary transformation to the helicity basis the noninteracting Hamiltonian (1) becomes $H_{0}=$ $\sum_{k, \alpha} \xi_{k}^{\alpha} c_{k \alpha}^{\dagger} c_{k \alpha}$, with the two helicities denoted by Greek indices $\alpha \in\{+,-\}$.

$$
\xi_{k}^{ \pm}=\epsilon_{k}-\mu \pm 2 v \sqrt{\sin ^{2} k_{x}+\sin ^{2} k_{y}}
$$

with $\epsilon_{k}$ the next-nearest-neighbor Hubbard model dispersion

$$
\epsilon_{k}=-2 t\left(\cos k_{x}+\cos k_{y}\right)-4 t^{\prime} \cos k_{x} \cos k_{y} .
$$

Here and henceforth we set the lattice spacing to unity. The eigenvectors for the helicity bands are

$$
\left|\eta_{ \pm}(\mathbf{k})\right\rangle=\frac{1}{\sqrt{2}}\left(\begin{array}{c}
1 \\
\pm e^{i \theta(\mathbf{k})}
\end{array}\right)
$$

with

$$
e^{i \theta(\mathbf{k})}=\frac{\sin k_{y}-i \sin k_{x}}{\sqrt{\sin ^{2} k_{x}+\sin ^{2} k_{y}}} .
$$

The spin orientations along the helicity bands are shown in Fig. 1. The operator mapping to the helicity basis is given by

$$
c_{k \pm}=\frac{1}{\sqrt{2}}\left(c_{k \uparrow} \pm e^{-i \theta(\mathbf{k})} c_{k \downarrow}\right) .
$$

The bare antisymmetrized Hubbard interaction term $V_{\text {int }}$ in the helicity basis becomes [26]

$$
\begin{aligned}
V_{\text {int }}= & \frac{U}{16} \sum_{\alpha \beta \gamma \delta} \sum_{k_{1} k_{2} k_{3} k_{4}} \delta_{k_{1}+k_{2}-k_{3}-k_{4}}\left(\alpha e^{-i \theta\left(\mathbf{k}_{1}\right)}-\beta e^{-i \theta\left(\mathbf{k}_{2}\right)}\right) \\
& \times\left(\delta e^{i \theta\left(\mathbf{k}_{4}\right)}-\gamma e^{i \theta\left(\mathbf{k}_{3}\right)}\right) c_{k_{1} \alpha}^{\dagger} c_{k_{2} \beta}^{\dagger} c_{k_{3} \gamma} c_{k_{4} \delta} .
\end{aligned}
$$
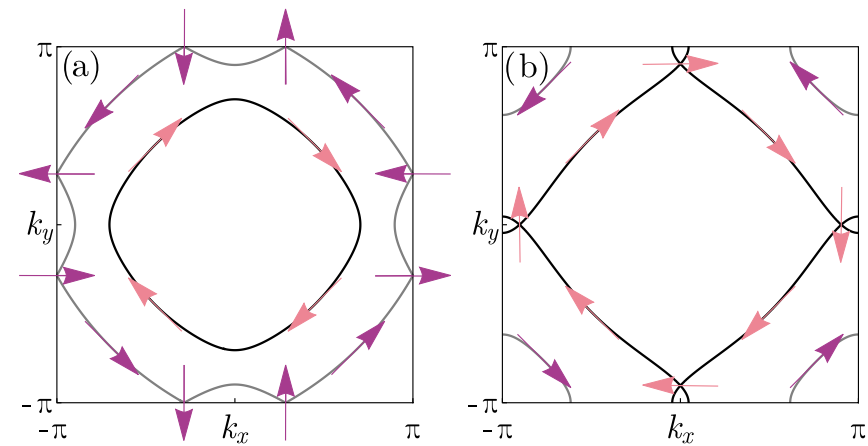

FIG. 1. (a) The two Fermi-surface sheets of the noninteracting Rashba-Hubbard model when the chemical potential is $\mu_{-}$, the value at which van Hove singularities occur on the outer sheet. (b) The same, but for the chemical potential $\mu_{+}$, the value at which van Hove singularities occur on the inner sheet. In both panels, arrows indicate the spin direction of the helicity eigenstate on that sheet of the Fermi surface. The parameters used are $t=1, t^{\prime}=0.3$, and $v=0.5$.

The two van Hove fillings are located at chemical potentials $\mu_{+}$and $\mu_{-}$, given by

$$
\begin{aligned}
\mu_{ \pm}= & \pm 2\left(-t+\frac{\left(t \pm 2 t^{\prime}\right)^{2}}{\sqrt{\left(t \pm 2 t^{\prime}\right)^{2}+v^{2}}}\right. \\
& \left.+v \sqrt{1-\frac{\left(t \pm 2 t^{\prime}\right)^{2}}{\left(t \pm 2 t^{\prime}\right)^{2}+v^{2}}}\right) .
\end{aligned}
$$

At each van Hove filling there are four van Hove points in the Brillouin zone, as shown in Fig. 1. The filling $\mu_{+}$is reminiscent of the scenario proposed by Yao and Yang [23], denoted a type-II van Hove singularity, with saddle points located away from the Brillouin zone edge. In the $\mu_{-}$case the type-I van Hove point [20-22] splits into two along the Brillouin zone edge. We call this the edge van Hove scenario. The van Hove points lie at $\mathbf{K}_{1,2}=\left(\mp \Pi^{+}, 0\right), \mathbf{K}_{3,4}=\left(0, \mp \Pi^{+}\right)$ for filling $\mu_{+}$and $\mathbf{K}_{1,2}=\left(\mp \Pi^{-}, \pi\right), \mathbf{K}_{3,4}=\left(-\pi, \mp \Pi^{-}\right)$for filling $\mu_{-}$, where

$$
\Pi^{ \pm}=\arccos \left(\mp \frac{t \pm 2 t^{\prime}}{\sqrt{\left(t \pm 2 t^{\prime}\right)^{2}+v^{2}}}\right) .
$$

The low-energy model that applies close to these van Hove fillings is given by the following imaginary time Lagrangian of spinless fermions:

$$
\begin{aligned}
\mathcal{L}_{ \pm}= & \sum_{a=1}^{4} \psi_{a}^{\dagger}\left(\partial_{\tau}-\xi_{a}^{ \pm}\left(-i \partial_{x},-i \partial_{y}\right)\right) \psi_{a}-\frac{g_{1}}{2} \psi_{a}^{\dagger} \psi_{\bar{a}}^{\dagger} \psi_{\bar{a}} \psi_{a} \\
& -\sum_{a=1}^{2} \sum_{b=3}^{4} g_{2} \psi_{a}^{\dagger} \psi_{b}^{\dagger} \psi_{b} \psi_{a}-\left[i g_{3} \psi_{4}^{\dagger} \psi_{3}^{\dagger} \psi_{2} \psi_{1}+\text { H.c. }\right]
\end{aligned}
$$

We retain only states around the van Hove points within the theory due to their enhanced contribution to the low-energy physics. This allows us to reduce the full Fermi surface to four regions with three possible interactions, depicted in Fig. 2. We number the patches as shown in the left hand panels of Fig. 2; 


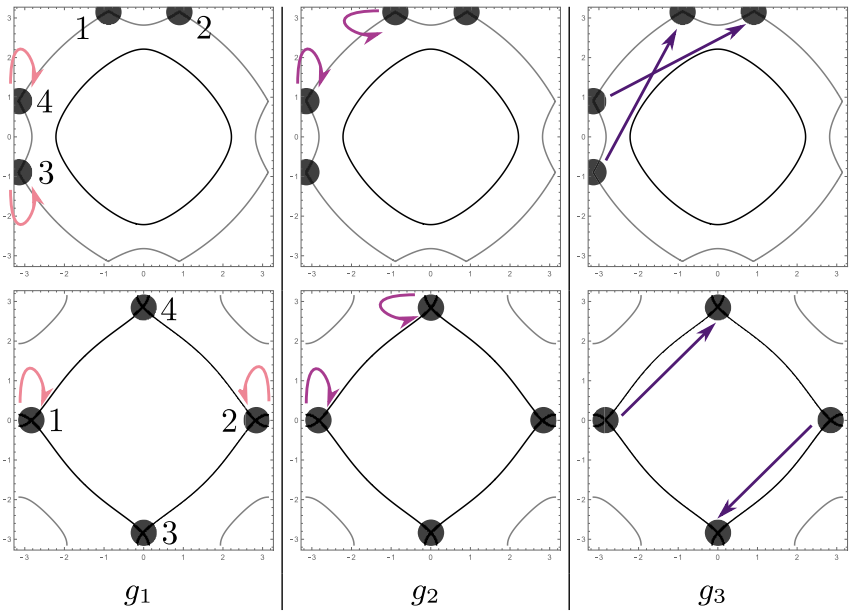

FIG. 2. Interactions allowed with four patches and spinless fermions. Fermi surfaces at chemical potential $\mu_{-}$(top row) and $\mu_{+}$(bottom row). $g_{1}$ and $g_{2}$ couplings denote density-density interactions between patches with zero and nonzero total momenta respectively. $g_{3}$ denotes an exchange interaction between all patches, conserving zero total momentum.

$\bar{a}$ denotes the patch with opposite momentum to patch $a$. The couplings $g_{i}$ are marginal at tree level, which justifies the application of one-loop RG to the weakly coupled problem. Density-density interactions on the same patch have a momentum prefactor and are therefore irrelevant and discarded from the effective field theory.

For the edge van Hove scenario there exist four inequivalent scattering vectors between patches. However, if we introduce four separate $g_{2}$ processes we find the couplings diverge equally and are indistinguishable during the flow. Therefore we describe the $g_{2}$ sector with one coupling for all scattering vectors.

The dispersions at the van Hove points are

$$
\begin{gathered}
\xi_{1,2}^{ \pm}(\mathbf{k})=-\frac{\delta k_{x}^{2}}{2 m_{x}^{ \pm}}+\frac{\delta k_{y}^{2}}{2 m_{y}^{ \pm}}, \quad \xi_{3,4}^{ \pm}(\mathbf{k})=-\frac{\delta k_{y}^{2}}{2 m_{x}^{ \pm}}+\frac{\delta k_{x}^{2}}{2 m_{y}^{ \pm}} \\
m_{x}^{ \pm}= \pm \frac{1}{\sqrt{\left(t \pm 2 t^{\prime}\right)^{2}+v^{2}}} \\
m_{y}^{ \pm}= \pm \frac{\sqrt{\left(t \pm 2 t^{\prime}\right)^{2}+v^{2}}}{t^{2} \pm 2 t t^{\prime}+v^{2}+t \sqrt{\left(t \pm 2 t^{\prime}\right)^{2}+v^{2}}}
\end{gathered}
$$

with $\delta k_{x}$ and $\delta k_{y}$ denoting the momentum relative to the van Hove saddle point value.

At van Hove filling, the density of states becomes logarithmic $\rho(\omega) \approx 2 \lambda^{ \pm} \ln (\Lambda / \omega)$ with $\Lambda$ an ultraviolet energy cutoff and $\omega$ the energy relative to the van Hove singularity. The constant $\lambda^{ \pm}=\sqrt{m_{x}^{ \pm} m_{y}^{ \pm}} / 4 \pi^{2}$ for filling $\mu_{ \pm}$. To determine the possible Fermi surface instabilities, the particleparticle and particle-hole susceptibilities are required. The susceptibilities that can have a double logarithmic divergence
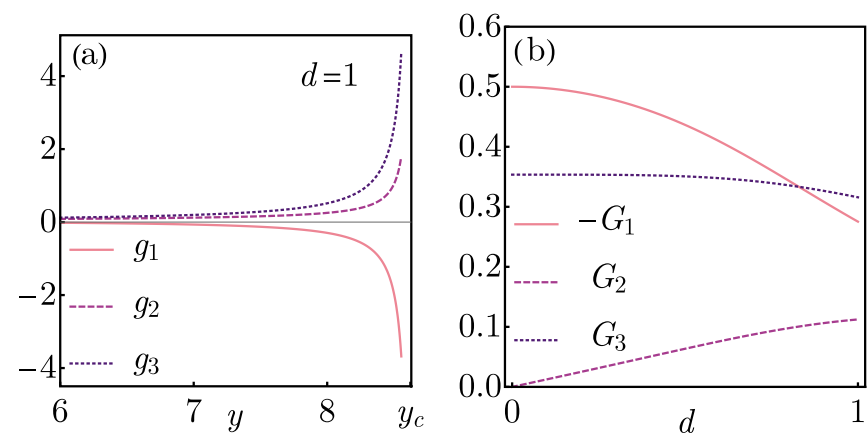

FIG. 3. (a) Nesting parameter $d=1$ numerical solution for flow of couplings $g_{i}$ with RG scale $y$ at $\mu_{+}$van Hove filling, starting from bare values $g_{i}(0)=0.04$. (b) Exponent values in (18) for $0 \leqslant d \leqslant 1$.

are [23]

$$
\chi_{0}^{\mathrm{pp}}(\omega) \approx \lambda^{ \pm} \ln ^{2}\left(\frac{\Lambda}{\omega}\right), \quad \chi_{\mathbf{q}_{2}}^{\mathrm{ph}}(\omega) \approx 2 \beta^{ \pm} \lambda^{ \pm} \ln \left(\frac{\Lambda}{\omega}\right),
$$

with

$$
\beta^{ \pm}=\frac{2 \sqrt{\kappa^{ \pm}}}{1+\kappa^{ \pm}} \ln \left|\frac{\kappa^{ \pm}+1}{\kappa^{ \pm}-1}\right| .
$$

The ratio $\kappa^{ \pm}=m_{y}^{ \pm} / m_{x}^{ \pm}$plays the role of a nesting parameter with the logarithm in $\beta^{ \pm}$diverging as $\kappa \rightarrow 1$ at perfect nesting. The complete set of relevant susceptibilities, including susceptibilities with single logarithmic divergences, are given in Appendix A.

\section{RESULTS}

We perform an RG analysis using $y=\ln ^{2}(\Lambda / \omega)$ as a flow parameter with $\Lambda$ a decreasing energy cutoff $[27,28]$. The flow equations for the dimensionless couplings $g_{i} \rightarrow \lambda^{ \pm} g_{i}$ are

$$
\begin{aligned}
& \dot{g}_{1}=-g_{1}^{2}-2 g_{3}^{2} ; \quad \dot{g}_{2}=d\left(g_{2}^{2}+g_{3}^{2}\right) ; \\
& \dot{g}_{3}=-2 g_{1} g_{3}+4 d g_{2} g_{3} .
\end{aligned}
$$

The $y$ dependence of the $g_{i}$ has been suppressed for brevity. $\dot{g}_{i}$ denotes the derivative $d g_{i} / d y$. We have discarded contributions with single logarithmic divergences in this picture; the full flow equations are given in Appendix B. $d \approx$ $d \chi_{\mathbf{q}_{2}}^{\mathrm{ph}}(y) / d \chi_{0}^{\mathrm{pp}}(y)$ is approximated as a constant nesting parameter $0 \leqslant d \leqslant 1$ to account for the additional logarithmic divergence at perfect nesting. At the beginning of the flow, $g_{i}(y=0)=\lambda^{ \pm} U$ with all couplings equal.

For $d=0$, the differential equations can be solved analytically with the critical value $y_{c}=(1+\sqrt{2}) / \lambda U$ for which the couplings diverge to strong coupling. We therefore use this as the cutoff for the phase transition. For $d \neq 0$, the critical value decreases and the $g_{3}$ coupling is enhanced. The solutions to the RG equations for $d=0$ and 1 are shown in Fig. 3. $g_{3}$ retains the sign of $g_{3}(0)$ due to the $\beta$ function vanishing as the coupling goes to zero. $g_{1}$ decreases under the RG and eventually becomes negative, leading to superconductivity.

The coupling constants $g_{i}$ flow to strong coupling as $y \rightarrow$ $y_{c}$, therefore our one-loop RG can only provide a qualitative picture of the phase diagram. We introduce the asymptotic 
form

$$
g_{i} \approx \frac{G_{i}}{y_{c}-y}
$$

to describe the divergence of the couplings [22].

To analyze the nature of the Fermi surface instabilities, we introduce infinitesimal test vertices for several possible types of order: superconductivity, $\mathbf{q}_{1}$ and $\mathbf{q}_{2}$ density waves, and Fulde-Ferrell-Larkin-Ovchinnikov (FFLO) superconductivity with finite momentum Cooper pairs. The resulting addition to the Lagrangian is

$$
\begin{aligned}
\delta \mathcal{L}= & \sum_{a=1}^{4}\left[\Delta_{a \bar{a}} \psi_{a}^{\dagger} \psi_{\bar{a}}^{\dagger}+\phi_{a \bar{a}} \psi_{a}^{\dagger} \psi_{\bar{a}}\right] \\
& +\sum_{a=1}^{2} \sum_{b=3}^{4}\left[\phi_{a b} \psi_{a}^{\dagger} \psi_{b}+\Delta_{a b} \psi_{a}^{\dagger} \psi_{b}^{\dagger}\right]+\text { H.c. }
\end{aligned}
$$

Spatially uniform $(\mathbf{q}=0)$ charge and magnetic orderings are suppressed due to the irrelevance of the intrapatch densitydensity interaction.

We find for the superconducting channel

$$
\left(\begin{array}{l}
\dot{\Delta}_{12} \\
\dot{\Delta}_{21} \\
\dot{\Delta}_{34} \\
\dot{\Delta}_{43}
\end{array}\right)=\left(\begin{array}{cccc}
-g_{1} & g_{1} & 2 i g_{3} & -2 i g_{3} \\
g_{1} & -g_{1} & -2 i g_{3} & 2 i g_{3} \\
-2 i g_{3} & 2 i g_{3} & -g_{1} & g_{1} \\
2 i g_{3} & -2 i g_{3} & g_{1} & -g_{1}
\end{array}\right)\left(\begin{array}{c}
\Delta_{12} \\
\Delta_{21} \\
\Delta_{34} \\
\Delta_{43}
\end{array}\right) \text {. }
$$

The dot again denotes a derivative with respect to $y$. The two possible nonzero eigenvalues of this matrix are $\varepsilon_{1}=-2\left(g_{1}-2 g_{3}\right)$ and $\varepsilon_{2}=-2\left(g_{1}+2 g_{3}\right)$ with corresponding eigenvectors $\mathbf{v}_{1}=(-i, i,-1,1)^{\mathrm{T}} / \sqrt{4}, \mathbf{v}_{2}=$ $(i,-i,-1,1)^{\mathrm{T}} / \sqrt{4}$. The superconductivity in the helicity basis is chiral/antichiral depending on the sign of $g_{3}$. We repeat the analysis in the FFLO and the $\mathbf{q}_{1}$ and $\mathbf{q}_{2}$ density wave channels to find all possible orders. The order parameters obey $\dot{\Delta}_{j}=\varepsilon_{j} \Delta_{j}$; the susceptibilities of the possible orders are $\chi_{j}(y) \sim\left(y_{c}-y\right)^{G_{j}}[22]$.

The exponents for superconductivity and $\mathbf{q}_{2}$ density wave orders are given by $G_{\mathrm{SC}_{1}}=2\left(G_{1}-2 G_{3}\right), G_{\mathrm{SC}_{2}}=2\left(G_{1}+\right.$ $\left.2 G_{3}\right), G_{\mathrm{DW} \pm}^{\mathbf{q}_{2}}=-d\left(G_{2} \pm 2 G_{3}\right)$. The FFLO superconductivity and $\mathbf{q}_{1}$ density wave order are suppressed and the $\mathbf{q}_{2}$ density wave order is also suppressed away from perfect nesting. The exponents for FFLO and $\mathbf{q}_{1}$ density wave order are $G_{\mathrm{FFLO}}=$ $2 d_{\alpha}\left(y_{c}\right) G_{2}, G_{\mathrm{DW}}^{\mathbf{q}_{1}}=-d_{\gamma}\left(y_{c}\right) G_{1}$ with $d_{\alpha}$ and $d_{\gamma}$ defined in Appendix B.

In order to obtain a picture of the RG flow to strong coupling we use the monotonically increasing $g_{2}$ as a flow parameter and redefine the remaining couplings $g_{1}=x_{1} g_{2}$ and $g_{3}=x_{3} g_{2}$ [24]. The flow equations in terms of these redefined couplings are

$$
\begin{aligned}
\frac{d x_{1}}{d \ln g_{2}} & =-x_{1}-\frac{x_{1}^{2}+2 x_{3}^{2}}{d\left(1+x_{3}^{2}\right)}, \\
\frac{d x_{3}}{d \ln g_{2}} & =-x_{3}-\frac{2 x_{3}\left(x_{1}-2 d\right)}{d\left(1+x_{3}^{2}\right)} .
\end{aligned}
$$

The fixed points of these equations describe four trajectories of the RG flow. The flow diagram is plotted in Fig. 4(a) with
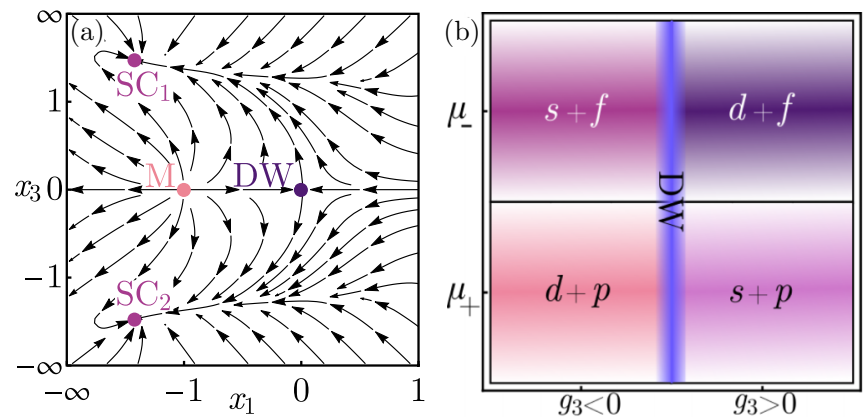

FIG. 4. (a) The RG flows of the couplings to log-squared accuracy and for nesting parameter $d=1$, projected onto the $x_{1} x_{3}$ plane, where $x_{1}=g_{1} / g_{2}$ and $x_{3}=g_{3} / g_{2}$. Fixed points in this diagram correspond to flow trajectories in the full space along which the ratios $x_{1}$ and $x_{3}$ become fixed. To include the points at infinity, the axes have been rescaled according to $x \rightarrow x /(1+|x|)$. M denotes the metallic trajectory, DW the $\mathbf{q}_{2}$ density wave trajectory, and $\mathrm{SC}_{1}$ and $\mathrm{SC}_{2}$ the superconducting trajectories. (b) Schematic phase diagram near the two van Hove fillings, corresponding to chemical potentials $\mu_{-}$and $\mu_{+}$, respectively. For the model (1), in which the on-site Hubbard interaction is repulsive, the coupling $g_{3}$ is always positive; however, for completeness, we have included in the phase diagram also negative values of $g_{3}$, which might arise as a result of competing interactions in more complicated microscopic models. The sign of $g_{3}$ determines whether the singlet component of the superconductivity is $s$ - or $d$-wave. Density-wave order dominates when $g_{3}$ is close to zero.

$d=1$. For the metallic fixed point $g_{2}$ does not flow. The density wave phase exists for $g_{3} \rightarrow 0$; in this case $g_{1} \rightarrow 0$ and only $g_{2}$ diverges. For the superconducting trajectories, all couplings diverge with ratios that depend on $d$. As $d \rightarrow 0$, the density wave and metal trajectories merge and only the metallic phase survives.

We now consider the superconducting order parameter in the original $\{\uparrow, \downarrow\}$ spin basis. We name the discrete order parameter in analogy to the continuum angular momentum channels. The even-parity order parameters are the isotropic $s$-wave channel $\Delta_{s}=\Delta(1,1,1,1)$ and nodal $d$-wave $\Delta_{d}=\Delta(1,1,-1,-1)$, where these four-component vectors give the phase of the superconducting order parameter at each of the four van Hove points, $\left(\mathbf{K}_{1}, \mathbf{K}_{2}, \mathbf{K}_{3}, \mathbf{K}_{4}\right)$. The odd-parity order parameters correspond to chiral $p$-wave $\Delta_{p_{x}+i p_{y}}=\Delta(-i, i,-1,1)$ and antichiral $p$-wave $\Delta_{p_{x}-i p_{y}}=$ $\Delta(i,-i,-1,1)$ in the Yao-Yang van Hove scenario but can represent higher-order angular momentum channels for the edge van Hove scenario. The continuum order parameter can be written as $\boldsymbol{\Delta}(\mathbf{k})=\left(\Psi_{s}(\mathbf{k})+\mathbf{d}(\mathbf{k}) \cdot \boldsymbol{\sigma}\right) i \sigma_{y}$. In the YaoYang scenario, the singlet component of the superconductivity $\Psi_{s}(\mathbf{k})$ corresponds to an $s$-wave form for $G_{\mathrm{SC}_{1}}$ and a $d$-wave form for $G_{\mathrm{SC}_{2}}$. The triplet component is helical and forms with chiral $p_{x}+i p_{y}$ superconductivity for one spin polarization and antichiral $p_{x}-i p_{y}$ superconductivity for the other, with $\mathbf{d}(\mathbf{k})=\left(\sin k_{x}, \sin k_{y}, 0\right)^{\mathrm{T}}$.

In the edge van Hove scenario the form factor is more complicated. If the van Hove points lie at $( \pm \pi / 2, \pi),(-\pi, \pm \pi / 2)$ along the Brillouin zone edge, $G_{\mathrm{SC}_{1}}$ corresponds to a superposition of singlet $d$-wave superconductivity with form factor $\Psi_{s}(\mathbf{k})=\cos 2 k_{x}-\cos 2 k_{y}$ and 
triplet $f$-wave superconductivity with the form factor $\mathbf{d}(\mathbf{k})=$ $\left(\cos k_{x}-\cos k_{y}\right)\left(\sin k_{x}, \sin k_{y}, 0\right)^{\mathrm{T}}$. The singlet component of $G_{\mathrm{SC}_{2}}$ is $s$-wave instead of $d$-wave. For van Hove points lying at different positions along the Brillouin zone edge the form factor requires higher harmonics, up to infinite order as the van Hove points approach the $(0, \pi)$ limit.

Additionally there exists a narrow window of density wave order for $g_{3} \rightarrow 0$. The competition between unconventional superconductivity and density-wave order has been seen to arise theoretically in similar spin-orbit split systems such as at oxide interfaces [11]. A schematic phase diagram is given in Fig. 4(b).

\section{SUMMARY AND DISCUSSION}

When the Fermi surface passes through saddle points in the band structure, the density of states is enhanced within the region of the saddle. This allows for an analytical treatment of the RG flow equations and an unbiased analysis of competing phases.

We have shown that mixed-parity superconductivity arises naturally in systems with antisymmetric spin-orbit coupling. The direction of the triplet $\mathbf{d}(\mathbf{k})$ vector is determined by the local spin quantization axis. Thus the triplet component of the superconducting order parameter forms a helical state, analogous to the quantum spin Hall insulator [29]. The helical superconductivity preserves time-reversal symmetry. The mixed-parity superconducting state is topologically nontrivial if the triplet component is greater than the singlet component [30,31]. Our case, where both components are equal, lies on the boundary between the topologically trivial and nontrivial phases. Our results suggest the superconductor can be tuned to a topological state, and could be useful for device applications and topological quantum computing.

Recently, we became aware of a related study on the hexagonal lattice [32].

\section{ACKNOWLEDGMENTS}

We thank J. Betouras and D. Efremov for useful discussions. M.J.T. acknowledges financial support from the CMCDT under EPSRC (UK) Grant No. EP/L015110/1. C.A.H. acknowledges financial support from the EPSRC (UK), Grant No. EP/R031924/1. He is also grateful to Rice University for a recent visiting position during which part of this work was completed.

\section{APPENDIX A: FULL PARTICLE-HOLE AND PARTICLE-PARTICLE SUSCEPTIBILITIES}

To compute the full RG flow equations including terms of single logarithmic divergence, all particle-particle and particle-hole susceptibilities are required.

The complete expressions, including susceptibilities reproduced from (15), are [23]

$$
\begin{aligned}
& \chi_{0}^{\mathrm{pp}}(\omega) \approx \lambda^{ \pm} \ln ^{2}\left(\frac{\Lambda}{\omega}\right), \quad \chi_{0}^{\mathrm{ph}}(\omega) \approx 2 \lambda^{ \pm} \ln \left(\frac{\Lambda}{\omega}\right) \\
& \chi_{\mathbf{q}_{1}}^{\mathrm{ph}}\left(\omega \approx 2 \gamma \lambda^{ \pm} \ln \left(\frac{\Lambda}{\omega}\right), \quad \chi_{\mathbf{q}_{2}}^{\mathrm{ph}}(\omega) \approx 2 \beta^{ \pm} \lambda^{ \pm} \ln \left(\frac{\Lambda}{\omega}\right),\right. \\
& \chi_{\mathbf{q}_{2}}^{\mathrm{pp}}(\omega) \approx 2 \alpha^{ \pm} \lambda^{ \pm} \ln \left(\frac{\Lambda}{\omega}\right) ; \\
& \alpha^{ \pm}=\frac{1+\kappa^{ \pm}}{2 \sqrt{\kappa^{ \pm}}}, \quad \beta^{ \pm}=\frac{2 \sqrt{\kappa^{ \pm}}}{1+\kappa^{ \pm}} \ln \left|\frac{\kappa^{ \pm}+1}{\kappa^{ \pm}-1}\right|
\end{aligned}
$$

The vectors $\mathbf{q}_{1}$ and $\mathbf{q}_{2}$ are given by $2 \mathbf{K}_{1}$ and $\mathbf{K}_{3}-\mathbf{K}_{1}$, respectively. The \pm signs of susceptibilities have been suppressed. $\gamma$ denotes an additional nesting parameter introduced by hand to suppress or enhance $\mathbf{q}_{1}$ scattering processes relative to the zero-momentum particle-hole processes [24].

\section{APPENDIX B: FULL RG FLOW EQUATIONS}

The complete flow equations including all single and quadratic logarithmic terms are

$$
\begin{aligned}
& \dot{g}_{1}=-g_{1}^{2}-2 g_{3}^{2}-2 g_{2}^{2} d_{1}+g_{1}^{2} d_{\gamma} ; \\
& \dot{g}_{2}=-2 g_{1} g_{2} d_{1}+\left(g_{2}^{2}+g_{3}^{2}\right) d_{\beta}-g_{2}^{2} d_{\alpha} ; \\
& \dot{g}_{3}=-2 g_{1} g_{3}+4 g_{2} g_{3} d_{\beta} .
\end{aligned}
$$

The derivative $\dot{g}_{i}=d g_{i} / d y$. The $d_{x}(y)$ parameters are defined as $d_{1}(y)=d \chi_{0}^{\mathrm{ph}}(y) / d \chi_{0}^{\mathrm{pp}}(y), d_{\gamma}(y)=d \chi_{\mathbf{q}_{1}}^{\mathrm{ph}}(y) / d \chi_{0}^{\mathrm{pp}}$ $(y), d_{\beta}(y)=d \chi_{\mathbf{q}_{2}}^{\mathrm{ph}}(y) / d \chi_{0}^{\mathrm{pp}}(y), d_{\alpha}(y)=d \chi_{\mathbf{q}_{2}}^{\mathrm{pp}}(y) / d \chi_{0}^{\mathrm{pp}}(y)$.

The functions $d_{x}(y), x=1, \alpha, \beta, \gamma$; have the asymptotic forms $d_{x}(y) \rightarrow 1$ as $y \rightarrow 0$ and $d_{x}(y) \rightarrow x / \sqrt{y}$, for $y \rightarrow \infty$. When solving the system of differential equations numerically we approximate the functions $d_{x}(y)$ by $d_{x}(y)=x / \sqrt{x^{2}+y}$ to interpolate between small- $y$ and large- $y$ asymptotic forms [23,24].
[1] S. D. Sarma, M. Freedman, and C. Nayak, npj Quantum Inf. 1, 15001 (2015).

[2] M. Sato and Y. Ando, Rep. Prog. Phys. 80, 076501 (2017).

[3] W. Kohn and J. M. Luttinger, Phys. Rev. Lett. 15, 524 (1965).

[4] M. Smidman, M. B. Salamon, H. Q. Yuan, and D. F. Agterberg, Rep. Prog. Phys. 80, 036501 (2017).

[5] L. P. Gor'kov and E. I. Rashba, Phys. Rev. Lett. 87, 037004 (2001).

[6] E. Bauer, G. Hilscher, H. Michor, Ch. Paul, E. W. Scheidt, A. Gribanov, Yu. Seropegin, H. Noël, M. Sigrist, and P. Rogl, Phys. Rev. Lett. 92, 027003 (2004).
[7] K. V. Samokhin, E. S. Zijlstra, and S. K. Bose, Phys. Rev. B 69 , 094514 (2004).

[8] M. Nishiyama, Y. Inada, and G.-Q. Zheng, Phys. Rev. Lett. 98, 047002 (2007).

[9] T. Yoshida and Y. Yanase, Phys. Rev. B 93, 054504 (2016).

[10] K. Takasan, A. Daido, N. Kawakami, and Y. Yanase, Phys. Rev. B 95, 134508 (2017).

[11] M. S. Scheurer and J. Schmalian, Nat. Commun. 6, 6005 (2015).

[12] Y. Lu, W.-Y. He, D.-H. Xu, N. Lin, and K. T. Law, Phys. Rev. B 94, 024507 (2016). 
[13] T. Yokoyama, S. Onari, and Y. Tanaka, Phys. Rev. B 75, 172511 (2007).

[14] K. Shigeta, S. Onari, and Y. Tanaka, J. Phys. Soc. Jpn. 82, 014702 (2013).

[15] A. Greco and A. P. Schnyder, Phys. Rev. Lett. 120, 177002 (2018).

[16] R. Ghadimi, M. Kargarian, and S. A. Jafari, Phys. Rev. B 99, 115122 (2019).

[17] X. Lu and D. Sénéchal, Phys. Rev. B 98, 245118 (2018).

[18] I. M. Lifshitz, Zh. Eksp. Teor. Fiz. 38, 1569 (1960) [Sov. Phys. JETP 11, 1130 (1960)].

[19] G. E. Volovik, Low Temp. Phys. 43, 47 (2017).

[20] H. J. Schulz, Europhys. Lett. 4, 609 (1987).

[21] P. Lederer, G. Montambaux, and D. Poilblanc, J. Phys. (Paris) 48, 1613 (1987).
[22] N. Furukawa, T. M. Rice, and M. Salmhofer, Phys. Rev. Lett. 81, 3195 (1998).

[23] H. Yao and F. Yang, Phys. Rev. B 92, 035132 (2015).

[24] R. Nandkishore, L. S. Levitov, and A. V. Chubukov, Nat. Phys. 8, 158 (2012).

[25] J.-Q. Huang, C.-H. Hsu, H. Lin, D.-X. Yao, and W.-F. Tsai, Phys. Rev. B 93, 155108 (2016).

[26] L. Wang and O. Vafek, Physica C 497, 6 (2014).

[27] R. Shankar, Rev. Mod. Phys. 66, 129 (1994).

[28] K. Le Hur and T. M. Rice, Ann. Phys. 324, 1452 (2009).

[29] X.-L. Qi, T. L. Hughes, S. Raghu, and S.-C. Zhang, Phys. Rev. Lett. 102, 187001 (2009).

[30] M. Sato and S. Fujimoto, Phys. Rev. B 79, 094504 (2009).

[31] C.-K. Lu and S. Yip, Phys. Rev. B 82, 104501 (2010).

[32] W. Qin, L. Li, and Z. Zhang, Nat. Phys. 15, 796 (2019). 